\title{
Hans-Ulrich Thamer
}

\section{Der öffentliche Umgang mit der Vergangenheit im deutschen und italienischen Nationalstaat}

Geschichtsbilder sind Medien der Selbstverständigung einer Gesellschaft. Die Konstruktion und Popularisierung von Geschichtsbildern sind geeignete Ressourcen für die Entfaltung politischer Identitäten und Normen mit dem Ziel der politisch-sozialen Integration. Sie transportieren Ideale und Zielvorstellungen, mit denen sich politisches Handeln rechtfertigen läßt und mit denen individuelle Loyalitäten und Interessen auf regionale und nationale Gemeinschaftsformen bezogen und verpflichtet werden sollen. Die Konstruktion und Vermittlung von Geschichtsbildern ist somit Teil eines Vergemeinschaftungsvorganges, in dem sich das Selbstverständnis einer Nation oder einer anderen politischen Ordnungsform konstituiert. Das gilt besonders für die Epoche der Nationalstaaten, die ihre Legitimation auf das historische Argument stützen ${ }^{1}$.

Unser Thema läßt sich auf vielfache Weise behandeln. Einmal als eine Rekonstruktion des nationalen Geschichtsdiskurses, d.h. als Frage nach dem Einsatz von Geschichte als Argument zur Legitimation der nationalen Gemeinschaft. Vergangenheit in diesem Kontext kann weit zurückliegende, zu einem Mythos geronnene Zeiten meinen, die in der Regel positiv konnotiert sind. Zum anderen können wir in einem engeren Sinne Vergangenheit als Frage nach dem politischen Umgang mit der besonders belastenden nationalsozialistischen bzw. faschistischen Vergangenheit der beiden postdiktatorialen Staaten Deutschland und Italien verstehen und folgen damit dem öffentlichen Sprachgebrauch vor allem in Deutschland, der Vergangenheit auf die Zeit der NS-Diktatur reduziert und die Erinnerung daran nach einer langen Phase der Tabuisierung fast zu einer negativen Identität der Deutschen macht. Doch widerspricht eine solche Reduktion der $\mathrm{Ge}$ schichtskultur auf die Zeit der beiden faschistischen Diktaturen allen geschichtswissenschaftlichen Erkenntnissen über den historischen Ort der beiden Faschismen, die eben keine Insel oder „Parenthese“ (De Felice) im Fluß der Geschichte

1 Vgl. dazu die Ergebnisse der neueren Nationalismus-Forschung. Zusammengefaßt bei $\mathrm{Ha}$ gen Schulze, Staat und Nation in der europäischen Geschichte (München 1994); Benedict Anderson, Die Erfindung der Nation. Zur Karriere eines erfolgreichen Konzeptes (Frankfurt a.M. 1987); Etienne François, Hannes Siegrist, Jakob Vogel (Hrsg.), Nation und Emotion. Deutschland und Frankreich im Vergleich (Göttingen 1995). 
des 19. und 20. Jahrhunderts darstellten und sich umgekehrt auch einem Bündel von Vergangenheitskonstruktionen zur Rechtfertigung der eigenen Herrschaft bedienten, die sich wiederum mit älteren, vor-faschistischen Bildern von $\mathrm{Ge}$ schichte berührten bzw. überschnitten. Überdies waren diese frühen nationalen Geschichtsbilder, die in die Faschismen hineingewachsen waren, nach 1945 nicht etwa verbraucht oder völlig diskreditiert, sondern sie lebten weiter und wurden im Sinne der These, daß es sich bei den Faschismen nur um eine Parenthese oder einen Betriebsunfall gehandelt habe, zur Anknüpfung an eine scheinbar unbeschädigte Vergangenheit revitalisiert. Die nationalen Mythen und Vergangenheitskonstruktionen, um die es vergleichend gehen soll, waren darum fester Bestandteil des politisch-gesellschaftlichen Prozesses der beiden Verfassungs- und Gesellschaftssysteme und ihres jeweils historisch begründeten Selbstverständnisses. Darum soll die Frage nach den Vergangenheitskonstruktionen als Kombination und Überlagerung der beiden skizzierten Ansätze behandelt werden. Überdies wird ein Vergleich der beiden politischen Kulturen und ihres Umganges mit der Vergangenheit angestrebt, um gemeinsame Grundmuster in der Konstruktion und Vermittlung von Geschichtsbildern, aber auch charakteristische Abweichungen oder Ungleichzeitigkeiten herauszuarbeiten, die einen Blick auf die jeweilige politische Kultur erlauben.

Die Repräsentation der Vergangenheit durch die Erzählung von Geschichte ist konstitutiver Bestandteil im Prozeß der Erfindung der Nation. Zwischen Nation und Geschichte, aber auch zwischen Nation und Mythos gibt es eine innere Verwandtschaft ${ }^{2}$. Die erzählte Vergangenheit soll den Nationen die Legitimation durch ihre vermeintlich lange Dauer sichern und ihre Gründung in eine weit zurückliegende Vergangenheit rücken bzw. Ereignisse dieser Vergangenheit in ein Kontinuum mit der jeweiligen Nationsbildung stellen. Die historische Begründung des Strebens nach Stiftung und Vollendung nationaler Einheiten führt zur Auswahl bestimmter Ereignisse und Begebenheiten, die in besonderer Weise die Einheit der Nation darstellen und begründen können.

Diese ausgewählten Bilder von der Vergangenheit, die durch verbale wie durch nicht-verbale Kommunikation vermittelt werden, verwandeln die historischen Ereignisse in Zeichen- und Deutungsangebote, lassen aus Fiktionen Normen entstehen. Das Reservoir dieser Bilder von Vergangenheit ist nicht unbegrenzt und wiederholt sich offenbar immer wieder. Das schafft Vertrautheit beim Publikum und verstärkt bzw. garantiert die Fähigkeit von Bildern, überhaupt ein Publikum zu erreichen. Was sich angesichts des begrenzten Repertoires an Bildern von Vergangenheit ändert, sind nicht die Ereignisse, die beschworen werden, wohl aber die Deutung und die Funktion, die sie in einem jeweiligen Kontext annehmen.

2 Etienne François, Hagen Schulze, Das emotionale Fundament der Nationen, in: Monika Flacke (Hrsg.), Mythen der Nationen. Ein europäisches Panorama. Eine Ausstellung des Deutschen Historischen Museums unter der Schirmherrschaft von Bundeskanzler Dr. Helmut Kohl (Berlin 1998) 17-32; im folgenden zitiert: Mythen der Nationen. 
Die Erzählung von Geschichte richtet sich auf Themen und Ereignisse von Mittelalter und Neuzeit, die ihren Erinnerungswert dadurch behalten, daß sie sich auf ein Normengefüge einer Gemeinschaft beziehen lassen. Mit der Entstehung von Nationalbewegungen und Nationalstaaten ist die Erzählung von Vergangenheit in der Regel auf die Nation bezogen und damit auf die Schaffung von nationalen Zeichen und Symbolen. Mit dem Wandel und den Brüchen der politischen Kulturen können die nationalen Bilder von Vergangenheit sich überlagern, aber sich auch in einem vielschichtigen Prozeß ablösen. Sie können auch miteinander konkurrieren, wenn es innerhalb einer Gesellschaft divergente bzw. antagonistische Auffassungen über die Legitimität der politisch-sozialen Ordnung gibt. Die Repräsentation von Vergangenheit kann dann dazu dienen, einer bestimmten Sicht der sozialen und politischen Welt Anerkennung zu verschaffen ${ }^{3}$. Dabei kann es zu Symbolkämpfen kommen ${ }^{4}$. Beispiele für symbolische Kämpfe zwischen den verschiedenen politischen Teilkulturen um die kulturelle Deutungsmacht finden sich im Deutschen Kaiserreich ebenso wie im Liberalen Italien zwischen 1870/71 und 1914 , noch sehr viel ausgeprägter und unvermittelt nebeneinander stehend etwa in der Weimarer Republik. Daneben kann es zu Ablösungen und Überlagerungen von Bildern durch historische Umbrüche kommen. Eine solche Zäsur in beiden Nationalstaaten bedeutet der Untergang der beiden national-faschistischen Führerdiktaturen des Dritten Reiches bzw. des Staates Mussolinis, die beide in nationalistisch-imperialer Übersteigerung an das überlieferte Reservoir der Nationalbilder anknüpften und nach $1945 \mathrm{zu}$ Neuorientierungen oder zu Umdeutungen in der historischen Begründung nationaler Identität herausforderten. Welche Vergangenheit sollte in den beiden postfaschistischen Gesellschaften rekonstruiert und erzählt werden? Die nationale Erzählung war durch ihre Instrumentalisierung für die Eroberungs- und Vernichtungspolitik diskreditiert und konnte nach 1945 auf der öffentlichen Ebene nur durch eine Art normativer Abgrenzung erzählt werden, indem nur bestimmte Teilbereiche der nationalen Tradition aufgegriffen wurden, wie etwa der Antifaschismus, oder indem andere scheinbar unbeschädigte Traditionen wieder aufgenommen wurden, die nach verbreiteter Meinung nicht vom Nationalsozialismus bzw. vom Faschismus okkupiert und korrumpiert waren.

Eine wichtige Rolle bei der Vermittlung von Vergangenheit zum Zwecke der nationalen Mobilisierung und Integration spielen die Medien, die dafür eingesetzt werden. Dazu gehören Schulbücher, historische Romane und Sachbücher, Feste und Feiern, Malerei und Denkmäler, Museen und Ausstellungen, Musik, Theater und Film. Sie haben eine je unterschiedliche soziale Reichweite und schichtenspezifische Wirkungsmöglichkeit. Die Intensität ihrer Botschaften nimmt durch Ver-

3 Grundsätzliche Überlegungen und Beispiele dafür bei Manuel Borutta, Die Kultur des Nationalen im Liberalen Italien. Nationale Symbole und Rituale in Rom 1870/71 und 1895, in: Quellen und Forschungen aus italienischen Archiven und Bibliotheken (QFIAB) 79 (1999) 480-529, 485 f., im folgenden zitiert: Borutta, Kultur des Nationalen.

4 Pierre Bourdieu, Sozialer Raum und soziale Macht, in: Pierre Bourdieu, J. C. Passeron, Rede und Antwort (Frankfurt a.M. 1992) 149. 
netzung und Multimedialität zu und ist überdies von zeitspezifischen Wahrnehmungsformen bestimmt. Es sollen für die erste Untersuchungsphase, d.h. für das deutsche Kaiserreich und den liberalen Staat Italiens bis zum Ersten Weltkrieg vor allem Zeugnisse der bildenden Kunst, Malerei und der Graphik herangezogen werden, die neben den Denkmälern zu den wichtigeren zeitgemäßen Formen nationaler Ikonographie gehörten. Bezogen auf ihre Trägerschichten waren sie vor allem zentraler Bestandteil der politisch-kulturellen Selbstverständigung des Bürgertums ${ }^{5}$. Daneben gab es populärere Medien, die auch für ein breiteres Publikum zugänglich waren und deren Analyse auch den Weg zu einer sozialgeschichtlich differenzierten Rezeptionsgeschichte eröffnen kann. Dazu gehören Feste und Feiern, populäre Druckerzeugnisse wie Bilderbögen oder Panoramen. Für die Zwischenkriegszeit sollen Ausstellungen als Erinnerungsorte auf Zeit und als Bedeutungsträger ausgewählt werden, die im Zeitalter neuer audiovisueller Medien dem Anspruch einer Demokratisierung der Bilderwelten und ihrer Vermittlung am besten entsprachen und die an die Stelle der mittlerweile stark diskreditierten Historienmalerei traten ${ }^{6}$. Für die beiden auf Massenmobilisierung und Massenkonsens zielenden national-faschistischen Bewegungen und Diktaturen kommen als charakteristische Ausdrucks- und Vermittlungsformen zusätzlich politische Massenrituale hinzu, die sich in aufdringlicher Form historischer Versatzstücke bedienten und Symbole und Zeichen aus den verschiedensten Herkunftsbereichen integrierten ${ }^{7}$. Für die zweite Nachkriegszeit bietet sich eine noch größere Bandbreite von Vermittlungstechniken und Formen an, von der klassischen Form der Literatur und Publizistik bis hin zu den modernen visuellen und elektronischen Massenmedien.

Folgt man dem Befund der Berliner Ausstellung "Mythen der Nationen“ (1998), so ist es jeweils ein Bündel von Ereignissen, die in den verschiedenen Medien als Hauptthemen der Erinnerungskultur vermittelt werden. Es sind im deutschen nationalen Diskurs fünf Ereignisse, die immer wiederkehren: die Schlacht im Teutoburger Wald ( $9 \mathrm{n}$. Chr.), der Tod des Stauferkönigs Friedrich Barbarossa (1190), die Verbrennung der Bannandrohungsbulle durch Martin Luther (1520), die Völkerschlacht bei Leipzig (1813) und die Kaiserproklamation in Versailles $(1871)^{8}$. Die Botschaft, die von diesen Schlüsselereignissen in der nationalen Geschichtserzählung ausging, war einfach: Arminius, der Befreier Germaniens, wurde zur Symbolfigur im Kampf gegen das Welsche, Westliche. Er fand sich im

$5 \mathrm{Vgl}$. Frank Becker, Bilder von Krieg und Nation. Die Einigungskriege in der bürgerlichen Öffentlichkeit Deutschlands 1864-1913 (München 2001), im folgenden zitiert: Becker, Bilder von Krieg und Nation.

6 Dazu jetzt zusammenfassend Christoph Kivelitz, Die Propagandaausstellung in europäischen Diktaturen. Konfrontation und Vergleich: Nationalsozialismus in Deutschland, Faschismus in Italien und die UdSSR der Stalinzeit (Bochum 1999).

7 Hans-Ulrich Thamer, Politische Rituale und politische Kultur im Europa des 20. Jahrhunderts, in: Jahrbuch für Europäische Geschichte 1 (2000) 79-97.

8 Monika Flacke, Die Begründung der Nation aus der Krise, in: Mythen der Nation 101128. 
heroischen Gestus auf Gedenkmünzen, auf Schmuck und Gedenkblättern, auf Ölgemälden und auf Denkmalsentwürfen, bis er schließlich auf einer Berghöhe bei Detmold eine ikonographisch endgültige Form annahm. An den im Kyffhäuser schlafenden Kaiser Barbarossa knüpfte sich die Erwartung auf Wiederkehr der Reichsherrlichkeit. Die Verbindung des nationalgesinnten schlafenden Kaisers mit monarchischen Symbolen brachte u.a. ein Kupferstich von Wilhelm von Kaulbach ${ }^{9}$ zum Ausdruck, aber auch im Kyffhäuser-Denkmal10 in Thüringen gehen der erwachende Barbarossa und der sich über ihm befindliche Wilhelm I. zu Pferde eine national-monarchische Symbiose ein. Wie weit der Barbarossamythos reichte, zeigte die Verwendung durch Adolf Hitler, der den Angriff auf die Sowjetunion im Sommer 1941 als „Unternehmen Barbarossa" tarnte. Im Lutherbild des 19. Jahrhunderts schließen sich gleich mehrere Deutungsstränge zusammen: der Freiheitsheld der Aufklärung, der Stifter einer Nationalreligion, die Verkörperung bürgerlicher Tugenden. Neben zahlreichen Gemälden und Stichen war es in diesem Fall das Wartburgfest vom 18. Oktober 1817, das als erstes Nationalfest das Lutherbild wirkungsvoll verbreitete und auf diese Weise eine politische Figur schuf, die Protestantismus und deutsche Nationswerdung miteinander verknüpfte. So fand sich Luther am Ende des 19. Jahrhunderts in der Gesellschaft von Arminius bis Bismarck unter der deutschen Eiche und auf den Sammeltassen.

Auch in den Bildern von dem Befreiungskrieg verbinden sich drei ikonographische Traditionen: 1. die Darstellung der Opferbereitschaft des gesamten Volkes, 2. das Aufgebot der Freiwilligen und 3. deren Einsegnung. Bei Adolf Menzel in seiner Lithographie "Viktoria" von $1836^{11}$ werden der Sieg und das Opfer der Toten bzw. Verwundeten für das Vaterland gefeiert. Bei Menzel geschah dies in einer bürgerlich-egalitären Form ohne die Darstellung von Hierarchien; in einem Schlachtengemälde von Johann Peter $\mathrm{Krafft}^{12}$ wurden Feldherren zu Pferde deutlich hervorgehoben und damit soziale Rangunterschiede im monarchisch-aristokratischen Sinne betont. Noch einmal wurde 1913 mit dem Völkerschlachtdenkmal in Leipzig der Befreiungskrieg zum Thema, diesmal durch die ästhetische Gestaltung der Monumentalität und Blockhaftigkeit als Aufruf zur nationalen Sammlung und Geschlossenheit. Für das vierte Thema des nationalen Diskurses, die Kaiserproklamation und Reichsgründung in Versailles, gibt es ein alles beherrschendes Bild, die Kaiserproklamation von Anton von Werner, eine Auftragsarbeit mit offiziellem Charakter, das bald auch für museale Zwecke, nämlich für

9 Wilhelm von Kaulbach, Friedrich Barbarossa im Kyffhäuser, 1841, Abb. in: Mythen der Nation 109.

10 Vgl. Gunther Mai, „Für Kaiser und Reich“. Das Kaiser-Wilhelm-Denkmal auf dem Kyffhäuser, in: ders. (Hrsg.), Das Kyffhäuser-Denkmal 1896-1996. Ein nationales Monument im europäischen Kontext (Köln, Weimar, Wien 1997) 149-178.

11 Adolph Menzel, Victoria!, 1836, Abb. in: Mythen der Nationen 118.

12 Jobann Peter Krafft, Siegesmeldung nach der Schlacht bei Leipzig, 1839, Abb. in: Mythen der Nationen 119. 
das Zeughaus in Berlin, nachgeschaffen wurde ${ }^{13}$. Es ist keine realistische Abschilderung der Ereignisse selbst, sondern eine symbolische Darstellung der politischen Kultur und Wertmuster des Kaiserreichs, das sich als eine festliche Versammlung von Fürsten und Generälen, als ein Heerlager und keineswegs eine Zivilgesellschaft darstellte. Anton von Werners Bild war der Ausgangspunkt für eine ganze Flut von Bildern, die alle die Verbindung von Bürgertum und Monarchie im Sinne des Nationalstaates propagierten und mit der Nationalisierung auch eine Militarisierung der bildungsbürgerlichen Schicht zum Ausdruck brachten bzw. verstärkten ${ }^{14}$. Die Verknüpfung der einzelnen nationalen Bilder und Symbole zu einer kontinuierlichen, aufeinander aufbauenden Nationalgeschichte, meist als einer Genealogie deutscher Ahnherren von Arminius über Luther zu Bismarck, wurde bald zu einem beliebten Thema vieler Blätter, die in Massenauflage reproduziert die Geschichte zur Vorgeschichte des Kaiserreichs umdeuteten.

Symbolische Gegenwelten neben oder gegen die Dominanz der monarchisch geprägten Nationalkultur gab es auch im autoritären Nationalstaat Bismarcks und Wilhelms. Das waren einerseits regionale Traditionen und historische Bezüge, wie sie etwa in Bayern gepflegt wurden, andererseits der Bezug auf Erinnerungsorte und -ereignisse, die quer zur herrschenden politischen Kultur standen, etwa die Erinnerung an die Revolution von 1848, an die die demokratische Emanzipationsbewegung erinnerte ${ }^{15}$. Eine Flucht in den geschichtslosen Ort der Natur als Antwort auf die monumentalisierte nationale Erinnerung an die Befreiungskriege im Jahr 1913 bei der Einweihung des Völkerschlachtdenkmals in Leipzig bedeutete das gleichzeitig stattfindende Treffen der Jugend- und Lebensreformbewegung im Herbst 1913 auf dem Hohen Meißner bei Kassel16.

Mittelalter und Risorgimento waren auch die Eckpunkte der italienischen nationalen Geschichtserzählung des 19. Jahrhunderts, angefangen mit der mythischen Überhöhung Dantes und seiner Umdeutung zum Propheten des Risorgimentos ${ }^{17}$. Zur Essenz des patriotisch gefärbten italienischen Geschichtsepos verdichtete sich die sogenannte sizilianische Vesper von 1282, ein Topos, der sich ebenso vorzüglich zur Behauptung des Unabhängigkeitswillens gegen die Fremdherrschaft eignete wie die nationale Deutung des „lombardischen Bundes“, des Sinnbildes für den vereinten Kampfeswillen gegen ausländische Bedrohung. Zum

\footnotetext{
13 Anton von Werner, Kaiserproklamation in Versailles, 1877, Abb. in: Mythen der Nationen 121.

14 Vgl. Becker, Bilder von Krieg und Nation, passim.

15 Vgl. Christina Klausmann, Ulrike Ruttmann, Die Tradition der Märzrevolution, in: Lothar Gall (Hrsg.), 1848. Aufbruch zur Freiheit. Eine Ausstellung des Deutschen Historischen Museums und der Schirn Kunsthalle Frankfurt a. Main zum 150jährigen Jubiläum der Revolution von 1848/49 (Berlin, Frankfurt a.M. 1998) 159-183.

16 Winfried Mogge, Jürgen Reulecke (Hrsg.), Hoher Meißner 1913. Der Erste Freideutsche Jugendtag in Dokumenten, Deutungen und Bildern (Köln 1988).

17 Dazu als Überblick Ilaria Porciani, „Fare gli italiani“, in: Mythen der Nation 199-222; Reinhard Elze, Pierangelo Schiera (Hrsg.), Italia e Germania. Immagini, modelli, miti fra due popoli nell'Ottocento: il Medievo. Das Mittelalter. Ansichten, Stereotypen und Mythen zweier Völker im neunzehnten Jahrhundert: Deutschland und Italien (Bologna, Berlin 1988).
} 
dramatischen Volksaufstand wurde der Aufstand in Genua 1746 stilisiert; die Bildsprache des frühen 19. Jahrhunderts erinnerte ganz an dramatische Appelle der französischen romantischen Revolutionsikonographie. Der Held der Handlung war ein Knabe namens Balilla. Er sollte nahtlos in die populäre Bildkunst Eingang finden bis hin zum Risorgimento und zum italienischen Faschismus. Von den Museen des Risorgimentos in der Regierungszeit Crispis bis hin zum Faschismus reicht die Wirkungsgeschichte dieses Mythos.

Zugleich schuf das Risorgimento seine ganz eigene Bild- und Erzähltradition, vor allem in der Beschwörung der Symbiose der demokratischen und monarchischen Einheitsbewegung in Gestalt des Treffens zwischen dem König und dem demokratischen Helden Garibaldi von Teano (1860) - ein italienisches Pendant zu dem kaiserlichen und preußisch-deutschen Reichsgründungsmythos, aber doch mit einem charakteristischen Unterschied: König und demokratischer Held begegnen sich ebenbürtig jeder auf dem Pferde, während Anton von Werners Heerlager allein das monarchisch-militärische Element hervorhebt ${ }^{18}$.

Im gemeinsamen Rückgriff auf die einheitsstiftende Erinnerung an das Risorgimento kam auch die Ambivalenz der italienischen Nationalkultur zum Ausdruck. Die symbolische Inbesitznahme von Rom als Hauptstadt am 3. Juli 1871 durch den König kopierte einerseits ein päpstliches Ritual des Amtsantrittes durch eine Prozession, an die nun ein festlicher Fackelzug zum Quirinalspalast als der neuen Residenz erinnerte, die einst Symbol der weltlichen Macht des Papstes war. Eine eigene national-monarchische Tradition und Legitimation wurde durch die gleichzeitige Berufung auf die Geschichte des Risorgimentos inszeniert: Auf der Piazza del Popolo wurden auf vierzehn großen Historiengemälden Schlüsselszenen des Risorgimentos von 1849 bis zum römischen Plebiszit von 1870 gezeigt, wobei die Geburt der Nation aus dem Krieg, ähnlich wie bei Anton von Werner, im Mittelpunkt stand. Vittorio Emanuele war der Held in der visuellen Erzählung der militärischen und politischen Aktionen, nur einmal tauchte Garibaldi als siegreicher Feldherr und damit als Konkurrent auf, der sich freilich in dieser Bildergeschichte dann beim Treffen von Teano dem Kommando des Königs unterwarf. Republikanische Erinnerungsorte und -figuren, wie etwa Mazzini und die Volksarmeen, blieben in dieser militärisch-monarchischen Bildwelt völlig ausgeblendet. In der Figur Garibaldis zeigte sich die vielfache Verwendbarkeit des Nationalhelden für eine politisch-soziale Identitätsstiftung, paßte er doch ebenso in das dynastische Geschichtsbild der Monarchie wie in die republikanische Tradition des Risorgimento, wie sie später vor allem von der dann regierenden Sinistra beschworen wurde. Auch für autoritäre Herrschaftsmodelle, etwa von Ministerpräsident Crispi, ließ sich der ehemalige Diktator von Sizilien in Anspruch nehmen ${ }^{19}$.

Die nationalen Bilder von der Vergangenheit, wie sie uns im 19. Jahrhundert in großen Historiengemälden und populären Bilderbögen, auf Denkmälern und in

18 Dazu Borutta, Kultur des Nationalen, bes. $500 \mathrm{ff}$.

19 Zum Gesamtzusammenhang Franz J. Bauer, Nation und Moderne im geeinten Italien, in: GWU 46 (1995) 16-31. 
Festinszenierungen begegnen, stiften in der Regel eine Verbindung, eine imaginäre Gemeinschaft von Vergangenheit und Gegenwart, indem sie dieser Gegenwart eine fiktive Vergangenheit oder Vorgeschichte entwerfen, die als gemeinsamer Bezugspunkt anerkannt wird: ein Akt der nationalen Einheit oder Befreiung, des nationalen Aufbruches oder Auferstehung. Die Geschichte bietet eine scheinbar schlüssige Antwort auf die Frage nach Herkunft, Legitimität und Bestimmung der zu konstituierenden nationalen Gemeinschaft. Diese Formierung einer imaginären Gemeinschaft basiert auf der Schilderung und Deutung einer Vergangenheit, die die Komplexität historischer Ereignisse auf eine essentielle Botschaft reduzierte, mithin einen Mythos entwirft, der in der Regel freilich wirkungsvoller war als jede historische Forschung und Darstellung, weil er einfache und sichere Aussagen versprach.

Der Begründung politischer Legitimität durch das historische Argument dienten auch die Geschichts- und Propagandaausstellungen der Zwischenkriegszeit, die sich allerdings teilweise auch der fachwissenschaftlichen Expertise und Rechtfertigung bedienten. Ausstellungen waren und sind Orte des ästhetisch-didaktischen Experimentes und reflektieren die veränderten Wahrnehmungsbedürfnisse der modernen Welt. Ihre Nähe zur Geschichts- und Kulturpolitik einer spezifischen nationalen Verfassungs- und Gesellschaftsordnung besteht seit ihren Anfängen. Ihre zunehmende Didaktisierung in der Zwischenkriegszeit ist Ergebnis zahlreicher Reformbemühungen seit der Volksbildungsbewegung der Jahrhundertwende. Zugleich knüpften sie an die wirtschaftlichen und sozialen Leistungsschauen an, wie sie sich in den nationalen und internationalen Industrie- und Gewerbeausstellungen, vor allem in den Weltausstellungen, publikumswirksam entwickelt hatten. Auch diese hatten sich zu ihrer Legitimation oft des historischen Argumentes bedient oder Produkte der bildenden Kunst als Symbol einer vergangenen ästhetischen Hochkultur zusätzlich eingesetzt.

Die politisch-integrativen Möglichkeiten, die in der Berufung auf eine gemeinsame Geschichte und einen gemeinsamen Gegner liegen und die eine moderne Präsentation durch eine Ausstellung publikumswirksam vermitteln könnte, suchte man auch in der von tiefen politisch-kulturellen Gegensätzen zerrissenen Weimarer Republik zu nutzen. National-politische Aufklärung sollte nach dem Willen der Veranstalter und auch in der Wahrnehmung des Publikums die historische Ausstellung zur Jahrtausendfeier des Rheinlandes 1925 in Köln leisten und dabei das Eigene vom Fremden, dem Französischen, abgrenzen bzw. herausstellen. Auch die Zugehörigkeit anderer Grenzregionen, nämlich des Saarlands und Danzigs, zum Reich sollten durch historische Ausstellungen im öffentlichen Bewußtsein befestigt werden. An diese Ausstellungstradition und -argumentation konnte die nationalsozialistische Geschichtspolitik, die von einer Vielzahl miteinander konkurrierender Institutionen mit großem Aufwand betrieben wurde, anknüpfen ${ }^{20}$. Im Winter 1933 wurde eine historische Ausstellung über den deut-

20 Hans-Ulrich Thamer, Geschichte und Propaganda. Kulturhistorische Ausstellungen in 
schen Osten präsentiert, die Ausstellung „Deutsches Volk - Deutsche Arbeit“ von 1934 deutete Geschichte ganz im Sinne überkommener nationaler Geschichtsbilder als eine Aufstiegs- und Niedergangsgeschichte, in der nun mit der „nationalsozialistischen Erhebung" ein neuer Aufbruch und eine Wiederanknüpfung an die historische Größe des Mittelalters und des friederizianischen Preußens gegeben war.

Der Zweck dieser Geschichtsausstellungen für die Repräsentation der Diktatur in Deutschland wie mit längerem Vorlauf in Italien war eindeutig: Es ging unter Anknüpfung an nationalistische historische Argumentationsmuster und moderne Ausstellungstechniken um die Verbreitung des Bildes einer geschlossenen nationalen Volks- und Leistungsgemeinschaft, die sich in die Kontinuität der jeweiligen Nationalgeschichte einfügte und den Anspruch erhob, deren Höhepunkt und Vollendung darzustellen. Das war nicht nur ein angeordnetes Bild, sondern entsprach den Erwartungen und Anpassungsbedürfnissen verschiedener Gruppen der Gesellschaft. Vermittelt wurde dieses Bild - und auch das kam beim Publikum an - mit den modernsten technischen audiovisuellen und ausstellungsästhetischen Mitteln, die ihrerseits Beweis für den vermeintlich fortschrittlichen Charakter des Regimes und seiner technisch-industriellen Modernität waren. Doch damit konnten sie zugleich ausgesprochen regressive, antiemanzipatorische und inhumane Inhalte transportieren. Aus der unterschiedlichen Gewichtung der historischen Argumente und der technisch-industriellen Elemente dieser Ausstellung lassen sich idealtypisch drei Formen von Ausstellungen herausarbeiten: erstens die historische Ausstellung im traditionellen Sinne, die meist Themen und Konzepte der nationalen Geschichtstradition aufgriff, zweitens die industrielle und soziale Leistungsschau, die sich auch des historischen Argumentes bediente, freilich meist zeitgeschichtlich ausgerichtet war und sich auf die faschistische Parteigeschichte als Leitthema konzentrierte, und drittens die reine Propagandaausstellung, die einzig und allein die Mythisierung der jeweiligen faschistischen Parteigeschichte betrieb.

Themen und Deutungsmuster der historischen Ausstellungen knüpften vor allem an das überkommene Reservoir nationalistisch-imperialer Geschichtsbilder an, denen wir bereits im Kaiserreich begegneten. Während die Titel der Ausstellungen diese scheinbar ungebrochene nationale Kontinuität suggerierten, wurde die eigentliche politische Botschaft meist durch Schlußsequenzen oder durch das Verfahren der Gegenüberstellung präsentiert und durch gestalterische Mittel verstärkt. Ausstellungen dieser Art trugen bezeichnenderweise Titel wie „Ewiges Deutschland“ (1934), „Das deutsche Antlitz im Spiegel der Jahrhunderte“ (1937) oder „Deutsche Größe“ (1940). Vergleichbar damit ist die italienische Ausstellung „Mostra Augustea della Romanità“ (1937/38) ${ }^{21}$. Es wurden jeweils Durchgänge

der NS-Zeit, in: GG 24 (1998) 349-381; im folgenden zitiert als Thamer, Geschichte und Propaganda.

21 Zum Vergleich Hans-Ulrich Thamer, Die Repräsentation der Diktatur. Geschichts- und Propagandaausstellungen im nationalsozialistischen Deutschland und im faschistischen Ita- 
durch die deutsche und italienische Geschichte angeboten, von der germanischen Vorzeit bis zum Nationalsozialismus oder vom römischen Imperium bis zum neuen faschistischen Imperium Mussolinis. Im Falle der Augustusausstellung wurde die Kontinuität bzw. Identität durch Parallelisierung erreicht ${ }^{22}$. So erschien Augustus als Feldherr und politischer Führer, aber auch als Schöpfer von Wohlstand und als Priester im Dienste einer Religion. Dies aber waren jene Qualitäten, die in der faschistischen Propaganda auch Mussolini unterstellt wurden. Die Parallelität der charismatischen Führerherrschaft und die Kontinuität nationaler Einheitsstiftung wurden in einem eigenen Saal der Wiedergeburt des Imperiums durch die Darstellung des nationalen Einigungswerkes Viktor Emanuels und Mussolinis inszeniert, die von antiken und modernen Triumphbögen eingerahmt waren. Eine Mussolinibüste durfte in deutlicher Parallelität zu Augustusbildnissen nicht fehlen. Inhaltlich orientierten sich die historischen Ausstellungen an traditionellen Geschichtsbildern des 19. Jahrhunderts, die nicht als spezifisch faschistisch oder nationalsozialistisch zu kennzeichnen sind, sofern darunter eine rassenideologische und national-revolutionäre, gewaltverherrlichende Umdeutung der nationalen Geschichtstraditionen zu verstehen ist.

Daß dies aber nicht die einzig denkbare und mögliche Geschichtsdeutung in den beiden Diktaturen war, die geschichts- und kulturpolitisch flexibel oder auch indifferent bzw. unausgeprägt genug war, um auch andere, nicht grundsätzlich abweichende national-konservative Deutungsmuster zu akzeptieren und propagandistisch zu nutzen, zeigen die erwähnten nationalgeschichtlichen Ausstellungen und die wenigen dazu publizierten Kataloge. Was dort zu lesen war oder in den Ausstellungen durch Inszenierungen und Ensemblebildung insinuiert wurde, waren herkömmliche Interpretationsmuster, die von nationaler Größe, von Machtstaat und Kampf bzw. Eroberung und Expansion handelten und oft von prominenten Historikern verfaßt waren. Im Falle der Augustusausstellung wich man hingegen vom zeitgenössischen Forschungsstand ab und orientierte sich an national-imperialen Geschichtsbildern, die in eine einfachere Kontinuitätslinie zum Staat Mussolinis zu bringen waren. Nicht Fälschung, sondern einseitige Akzentuierung war die Devise dieser Geschichtsinterpretation, während sich in das tradierte populäre nationalistische Geschichtsbild der NS-Ausstellungen immer mehr rassistische Interpretationen einschlichen. Darstellungsleitende Muster waren in der NS-Ausstellung meistens dichotomische Geschichtsbilder, etwa über die Machtentfaltung und Ausdehnung des Reiches einerseits, über die nationale Zersplitterung und den Verfall andererseits. Das Streben zum Reich, das seinen Höhe- und Endpunkt in der Entfaltung des Dritten Reiches fand, zog sich als roter Faden durch alle Ausstellungen. Rassenpolitische Motive bildeten nicht das eigentliche Ausstellungsthema, sondern dienten als Schlußsequenz einmal der

lien, in: Christof Dipper, Rainer Hudemann, Jens Petersen (Hrsg.), Faschismus und Faschismen im Vergleich. Wolfgang Schieder zum 60. Geburtstag (Vierow 1998) 229-246.

22 Dazu ausführlich Friedemann Scriba, Augustus im Schwarzhemd? Die Mostra Augustea della Romanità in Rom 1937/38 (Frankfurt a.M. 1995). 
Deutung des Machtverfalls in der bisherigen Geschichte und der Legitimation der neuen Politik des Dritten Reiches. In der Ausstellung „Nürnberg - die deutsche Stadt. Von der Stadt der Reichstage zur Stadt der Reichsparteitage“ (1937 im Germanischen Nationalmuseum) wurden in den Schlußsequenzen die Nürnberger Rassengesetze von 1935 in eine Kontinuitätslinie zur Vertreibung der Nürnberger Juden unter Karl IV. gestellt und damit die NS-Politik als Überwindung der „zersetzenden jüdischen Einflüsse“ präsentiert, die für den Machtverfall seit dem späten Mittelalter verantwortlich gemacht wurden, um dann die NS-Zeit als Wiederherstellung des Sinnes deutscher Geschichte zu feiern ${ }^{23}$.

Mit der Augustusausstellung in der Anlage und These durchaus vergleichbar war die Ausstellung "Deutsche Größe“ von 1940, die einen Höhepunkt nationalsozialistischer Ausstellungspolitik darstellte und aufgrund ihrer politischen Ausrichtung und ihrer Ausstellungsdidaktik in der NS-Presse auch entsprechend gefeiert wurde, da sie „zum ersten Mal ausschließlich unter politischen Gesichtspunkten konsequent durchgeführt und nicht mehr von halbherzigen Kompromissen eingeschränkt wurde" 24 . Die Themen waren dieselben wie in früheren Ausstellungen: deutsche Machtentfaltung im Mittelalter, Ostexpansion von Hanse und deutschem Orden, Aufstieg Österreichs und Brandenburg-Preußens und schließlich die Reichsgründung Bismarck, dazwischen immer wieder „innere Zersetzung“ und „zerstörerische Ideen“ als Gefährdung des Reiches. In dieser Linie des Auf und Ab bedeutete schließlich die Erlösergestalt Adolf Hitler die Verheißung auf Stabilität und Dauer. Er habe, wie der Historiker Fritz Rörig begeistert im Katalog formulierte, durch die „straffe Zusammenfassung des Reiches dem deutschen Erbe im Osten ungeahnte Möglichkeiten" 25 verliehen. In der Variante der Ausstellung, die im besetzten Belgien gezeigt wurde, baute man die NS-Europaideologie zusätzlich in die Argumentation ein. Optisch erfuhr die These von der Geschlossenheit eine Verstärkung durch eine einheitliche Raumgestaltung, die die Objekte als Bedeutungsträger auf optische Einheitlichkeit zurechtstutzte oder vergrößerte, indem man nicht mit dem authentischen Objekt, sondern mit Repliken arbeitete, die sich entsprechend verändern ließen.

Ich übergehe die Industrie- und Sozialausstellungen wie die Propagandaausstellungen, die zwar ausstellungstechnisch und didaktisch noch sehr viel aufwendiger und moderner waren und damit an die Standards heranreichten, die die italienische "Mostra della Rivoluzione Fascista" von $1932^{26}$ gesetzt hatte, die aber wie diese sich allein auf die Verherrlichung der faschistischen Parteigeschichte und des Führermythos konzentrierte und auf die Einordnung in die Geschichtstradi-

23 Belege bei Thamer, Geschichte und Propaganda 357.

24 Zit. ebd. 378.

25 Ausstellung Deutsche Größe. Unter der Schirmherrschaft des Stellvertreters des Fübrerreichsministers Rudolf $\mathrm{He} ß$ veranstaltet von der Dienststelle des Beauftragten des Führers für die Überwachung der gesamten geistigen und weltanschaulichen Schulung und Erziehung der NSDAP. Durchgeführt vom Amt für Schrifttumspflege (München 1940) 122.

26 Dazu Marla Stone, Staging Fascism. The Exhibition of the Fascist Revolution, in: Journal of Contemporary History 28 (1993) 215-243. 
tion verzichtete. Statt dessen setzte man auf die Verlockungen der Technik und der Massenzivilisation, die durch Ausstellungsdidaktik und -thematik den Modernitätsbeweis erbringen sollten.

Blicken wir abschließend auf den Umgang mit Geschichte in der zweiten Nachkriegszeit, so fällt grundsätzlich eine zunehmende Pluralisierung der Medien, der Themen und Deutungs- bzw. Aneignungsformen auf, die jede generalisierende Aussage erschwert. Gleichwohl gibt es trotz mancher abweichender Position einen Mainstream, der in charakteristischer Phasenverschiebung und Intensität in der Bundesrepublik Deutschland wie in Italien zu finden ist. Einen hier nicht weiter zu behandelnden Sonderfall stellt die DDR-Geschichtspolitik mit ihren verordneten Geschichtsbildern dar, die nach anfänglichen Parallelen mit zunehmender Stalinisierung und Behauptung einer Eigenstaatlichkeit einen eigenen Weg nahmen, der 1989 ein abruptes Ende ohne erkennbare Nachwirkung fand ${ }^{27}$.

Auf den ersten Blick war die Ausgangslage für das nationale Geschichtsbewußtsein in Deutschland und Italien nach 1945 offensichtlich durchaus deckungsgleich. Man war mit einem abrupten Bruch in der nationalen Geschichtskontinuität und den dort vorherrschenden Paradigmen konfrontiert. Die Geschichte war zwar nicht an ihr Ende gekommen, aber die "Geschichtsmelodie Deutschlands“, wie Theodor Heuß es formulierte ${ }^{28}$, war völlig zertrümmert. Nationale Geschichtsbilder, die durch die nationalsozialistische Hybris zur Rechtfertigung von Eroberung und Vernichtung mißbraucht worden waren und zu der These hypostasiert wurden, der Nationalsozialismus sei die Vollendung der deutschen Nationalgeschichte, waren zunächst völlig diskreditiert; Alternativen waren nur mühsam zu erkennen, da es aus der deutschen Gesellschaft auch kaum Widerstand gegen diese Deutungen und diese Herrschaft gegeben hatte. Eine „Entpreußung“ der deutschen Geschichte als Teil einer politischen Entnazifizierung war seitens der Besatzungsmächte zunächst angesagt. Der Nationalsozialismus galt in Umkehrung der positiven Deutung durch die Nationalsozialisten nun als logische Konsequenz der gesamten deutschen Geschichte, die von Luther über Friedrich den Großen und Bismarck bis Hitler führte und eine einzige „Misere“ darstellte. Allenfalls die Berufung auf die kulturellen Leistungen als „guten Kern“ der deut- . schen Geschichte, als Gegengewicht gegen die Geschichte des Macht- und Militärstaates blieb. Friedrich Meinecke, Altmeister der deutschen Geschichtswissenschaft, suchte einen Ausweg in der Verbindung von Geist und Macht und plädierte für den politisch-kulturellen Neuaufbau durch Goethe-Bünde.

Auch Italien stand 1945 vor einem Bruch in der nationalen Identitätsgeschichte, nachdem der Faschismus den Nationalgedanken völlig für sich vereinnahmt und die eigene Herrschaft unter breiter Zustimmung der italienischen Gesellschaft als Höhepunkt der italienischen Nationalgeschichte gedeutet hatte. Doch anders als

27 Aus der umfangreichen Literatur jetzt die knappe Zusammenfassung von Edgar Wolfrum, Geschichte als Waffe. Vom Kaiserreich bis zur Wiedervereinigung (Göttingen 2001) bes. 56146; für Italien Jens Petersen, Wandlungen des italienischen Nationalbewußtseins nach 1945 , in: QFIAB 71 (1991) 699-748.

28 Zit. nach Wolfrum, Geschichte als Waffe 57. 
in West-Deutschland besaß die Tradition der Resistenza, die in der eigenen Rhetorik und politischen Pädagogik der Nachkriegszeit sicherlich eine größere Bedeutung und Breite besaß als in der historischen Wirklichkeit unter der faschistischen Diktatur, eine große Bedeutung für die Wiedergewinnung einer nationalen Identität, die an die freiheitlich-demokratische Tradition des Risorgimento anzuknüpfen beanspruchte und sich als gemeinsamer Nenner für eine in sich heterogene Koalition aus Katholiken, Liberalen und Kommunisten anbot. „Die Resistenza wurde zum Gründungsmythos des neuen Staates und der neuen Gesellschaft" ${ }^{29}$, indem sie sich auf das Opfer der antifaschistischen Märtyrer und auf die Wiederbelebung des Aktes der nationalen Befreiung berief. Das bedeutete auch eine Befreiung von den Traditionen der nationalen Geschichte des Machtstaates und eine Hinwendung zur Geschichte der Gesellschaft, des Katholizismus, der Arbeiterbewegung, aber auch Unsicherheiten im Umgang mit der Geschichte der Nation und des Staates. Zugleich schützte der zum Mythos erstarrte Antifaschismus vor der Notwendigkeit einer historisch-empirischen Beschäftigung mit der faschistischen Vergangenheit. Indem der Faschismus zum Objekt eines tausendfach beschworenen Abscheus und einer rhetorischen Distanzierung wurde und blieb, brauchte man nicht über die historischen Zusammenhänge seines Entstehens und seiner Herrschaftsmechanik einschließlich des breiten Konsenses, den die Führer-Herrschaft Mussolinis gefunden hatte, nachzudenken und sich selbst unangenehme Fragen zu stellen. Die historische Aufarbeitung des Faschismus begann erst in den 1970er und 1980er Jahren, beispielsweise durch historische Streitschriften oder durch historische Ausstellungen über Kultur und Wirtschaft der 1930er Jahre. Damit waren die „Ferien von der Geschichte“, die 1945 eingesetzt hatten, zu Ende; es war die Möglichkeit eröffnet, „für die Massen ein kollektives Gedächtnis zurückzugewinnen “30.

Nationale Gegenmythen, die sich durch Widerstand gegen den Nationalsozialismus zusätzlich legitimiert hatten, gab es im Nachkriegsdeutschland nicht, sieht man einmal von dem verordneten Antifaschismus der DDR ab, der bis 1989 Politik und Gesellschaft des anderen deutschen Staates erlaubte, sich auf die Seite der Sieger zu stellen, wo man mehrheitlich doch zu den kleinen oder auch größeren Tätern bzw. Verantwortlichen gehörte ${ }^{31}$. Mit der Anerkennung staatlicher und sozialer Kontinuitätslinien über 1945 hinaus hat sich die Bundesrepublik den ungleich schwierigeren Part im Umgang mit der NS-Vergangenheit aufgeladen. Das erforderte eine schwierige Balance zwischen einer normativen politisch-moralischen Abgrenzung vom Nationalsozialismus einerseits und einer Integration der Millionen von Individuen andererseits, die das Dritte Reich als Mittäter bzw. Mitläufer oder auch in innerer Distanz überlebt und erlebt hatten, in die Staatsbürgergesellschaft der Bundesrepublik. Auch wenn dieser Prozeß im Rückblick trotz

29 Petersen, Wandlungen 711.

30 Ebd. 737.

31 Aus der umfangreichen Literatur jetzt Peter Reichel, Vergangenheitsbewältigung in Deutschland. Die Auseinandersetzung mit der NS-Diktatur von 1945 bis heute (München 2001). 
der vielen Widersprüchlichkeiten und Skandale im einzelnen als insgesamt gelungen bezeichnet werden kann, können die hohen politisch-moralischen Kosten dieses Integrationsvorganges, zudem es grundsätzlich keine Alternativen gab, nicht übersehen werden.

Geschichtspolitisch entsprach dieser ambivalenten politischen Strategie der Versuch, die „schlechte“ Tradition des Dritten Reiches von den unbeschädigten, positiven Traditionslinien der deutschen Nationalgeschichte zu trennen, was im Konkreten immer wieder zum Streit darüber führte, was zu diesem positiven Teil $\mathrm{zu}$ rechnen war und was nicht. Gehörten das Bismarck-Reich, die Traditionen Preußens dazu, oder sollte man trennen zwischen einem humanistischen, rechtstaatlichen Erbe und einer obrigkeitsstaatlich-militaristischen Entwicklungslinie und ihrem Weiterwirken andererseits. Wie sollte man mit dem Nationalgedanken umgehen, der nicht nur durch die nationalsozialistische Eroberungs- und Vernichtungspolitik aufs Schwerste belastet war, sondern dem durch die deutsche Teilung auch der territoriale Bezugspunkt fehlte.

Eine Antwort auf dieses schwere Erbe bestand im Verdrängen der Erinnerung an die NS-Zeit und auch in der Flucht aus der Geschichte. Die politischen Anknüpfungspunkte für eine Identitätsvergewisserung der bundesrepublikanischen politischen Kultur lagen in der Betonung gegenwärtiger und zukünftiger Aufgaben, im Aufbau eines Verfassungsstaates und eines funktionierenden parlamentarisch-demokratischen Systems, das die Fehler von Weimar nicht wiederholen und das sich grundsätzlich von der zweiten deutschen Diktatur der SED in der DDR abheben sollte. Der Versuch, die Erinnerung an den gescheiterten Volksaufstand in der DDR vom 17. Juni 1953 zu einem nationalen Gedenk- und Feiertag zu machen $^{32}$, scheiterte nicht nur an Gewohnheiten der bundesdeutschen Freizeitgesellschaft, sondern auch an den konträren Geschichtsdeutungen und -bildern, die sich mit dem 17. Juni verbanden. War es ein Arbeiteraufstand, in der Tradition der demokratischen Arbeiterbewegung, ein Volksaufstand mit dem Ziele einer Befreiung von der SED-Diktatur oder ein Aufstand für die Wiedervereinigung? Die Schwierigkeiten einer konsensualen historischen Einordnung spiegeln auch die Wandlungen in der bundesdeutschen Geschichtskultur. Mit dem Verblassen des antitotalitären Konsenses, der sich von Nationalsozialismus und Stalinismus gleichermaßen abgrenzte, verlor der 17. Juni seine Wirkungskraft als nationaler Feiertag, wie er anfänglich noch an nationalen Erinnerungsorten wie etwa dem Hermannsdenkmal oder in Erinnerungsreden an die Befreiungskriege begangen wurde; es traten allmählich verfassungspatriotische Verbindungen zur Revolution von 1848/49 oder auch zur demokratischen Arbeiterbewegungstradition in den Vordergrund. Die Freiheitsidee wurde im geschichtspolitischen Diskurs der Bundesrepublik mächtiger und suchte sich andere Anknüpfungspunkte außerhalb der etablierten nationalen Traditionsbilder. Das waren teilweise verschüttete Traditionen, die mitunter etwas krampfhaft wiederbelebt wurden, die aber für die poli-

32 Dazu Edgar Wolfrum, Geschichtspolitik in der Bundesrepublik Deutschland. Der Weg zur bundesrepublikanischen Erinnerung 1948-1990 (Darmstadt 1999). 
tisch-kulturelle Westorientierung der Bundesrepublik anschlußfähiger waren. Dazu berief man sich in der offiziellen Geschichtspolitik zunehmend auf die Traditionen der Paulskirche und anderer demokratischer Emanzipationsbewegungen, die aber nur langsam für eine breitere Öffentlichkeit attraktiv und in ihr historisch-politisches Bewußtsein integrierbar und rezipierbar wurden.

Der nationalen Erinnerung, in die diese demokratischen Traditionen eingepflanzt werden sollten, ging eine regionale Identitätsvergewisserung voraus, die am Anfang der Wiedergewinnung von Geschichte in den 1970er Jahren stand. Landesausstellungen über mittelalterliche Herrscherfiguren und Dynastien, die zu Begründern einer regionalen Identität erklärt wurden, machten den publikumswirksamen Anfang, begleitet von einem breiten kulturellen Bedürfnis nach Herkunftsvergewisserung, wie sich u.a. in der Denkmalschutzbewegung und der Rettung von erhaltenswerten Kulturgütern vor der Abrißbirne der Massenwohlstandsgesellschaft äußerte ${ }^{33}$. Im Unterschied zu Italien hat dieses Bemühen um regionale Identitätsstiftung keine zentrifugale Kraft entwickelt, die zu einer Gefährdung der nationalen politischen Integration und der sozial-ökonomischen Solidarität zu werden droht. Statt dessen wurden die Erfolge dieser Ausstellungen, zuletzt auch der „Preußen“-Ausstellung in Berlin, Anlaß zu Überlegungen, ein deutsches Geschichtsmuseum zu gründen. Wie kontrovers dieser Gedanke, der vor allem von der Bundesregierung Helmut Kohls nach dem politischen Wechsel 1982 aufgegriffen wurde, tatsächlich war, zeigen die heftigen öffentlichen Debatten, die auch zu Debatten um eine nationale Identität wurden ${ }^{34}$.

Die regionale Selbstvergewisserung durch den Rückgriff auf die Erinnerung an Mittelalter und frühneuzeitliche Staaten hat sich aber auch nicht gegen einen anderen, immer breiter werdenden geschichtspolitischen Trend gerichtet, obwohl dies von dem einen oder anderen Protagonisten dieser Rückkehr zur Geschichte vielleicht intendiert war. Gemeint ist der immer breiter werdende Anteil der Auseinandersetzung mit der NS-Vergangenheit, die seit den späten 1970er Jahren einsetzte und seither ungebrochen ist. Neue Identitätsdebatten in der Bundesrepublik, vor allem in den 1980er und 1990er Jahren, drehten sich um den Stellenwert des Nationalsozialismus im deutschen Politik- und Staatsverständnis, um die Verstrickung der deutschen Gesellschaft in die NS-Verbrechen, um die Verdrängung der entsprechenden Erinnerungs- und Schulddiskussion. Über Ergebnis und Folgen dieser teilweise erregten geschichtspolitischen Streitfälle gibt es nach wie vor unterschiedliche Einschätzungen: Die Erinnerung an eine „Vergangenheit, die nicht vergehen will“, und die der deutschen Öffentlichkeit einen „Erinnerungsimperativ“ (Wolfrum) auferlegte, hat nach der einen Überzeugung sich zum alles beherrschenden Grundzug einer rein negativen Identität der deutschen Gesellschaft entwickelt, die zu einer Lähmung und Starre führen könnte; sie hat sich nach Mei-

33 Hans-Ulrich Thamer, Vom Heimatmuseum zur Geschichtsschau. Museen und Landesausstellungen als Ort der Erinnerung und der Identitätsstiftung, in: Westfälische Forschungen 46 (1996) 429-448.

34 Als Dokumentation der Kontroverse Christoph Stölzl (Hrsg.), Deutsches Historisches Museum. Ideen - Kontroversen - Perspektiven (Berlin 1988). 
nung der linksliberalen Öffentlichkeit zu einem unverzichtbaren Bestandteil der politischen Kultur entwickelt, dessen Auflösung verhängnisvoll wäre. Freilich gilt, daß die Historisierung des Nationalsozialismus, die noch im Historikerstreit von 1986/87 als eine Gefährdung einer demokratischen Geschichtskultur galt, durch den Gang der Ereignisse, vor allem durch die politische Zäsur von 1989/90, längst im Gang ist und damit eine neue Phase des Umganges mit Vergangenheit eingeleitet hat, die in das 21. Jahrhundert weist. 